$\mathbf{M} \mathbf{S}$

médecine/sciences $1986 ; 2$ : 540-1

\title{
MÉDECINE/SCIENCES, SES LECTEURS ET SES PROJETS
}

\section{Axel Kahn \\ Rédacteur en chef, Paris Directeur de l'Unité de génétique et de pathologie moléculaires, Inserm U 129, CHU Cochin-Port- Royal, 24, rue du Fau- bourg Saint-Jacques, 75014 Paris.}

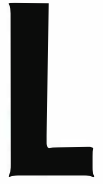

es " traditions " s'acquièrent rapidement à médecine/sciences. Le dernier numéro de l'année est donc traditionnellement pour nous l'occasion de faire un bilan de notre activité et de vous informer de nos projets.

En ce qui concerne notre lectorat et son jugement sur médecine/sciences, un questionnaire a été adressé à nos lecteurs européens au mois de mars 1986 ; parallèlement, une enquête a été faite dans le même sens au Québec. En France, les lecteurs appartiennent principalement à trois groupes dont chacun représente 20 à $25 \%$ du total : les médecins hospitaliers, les chercheurs en biologie et les étudiants, principalement ceux du troisième cycle. Le quart restant se distribue entre les médecins du secteur privé, diverses professions de santé et les enseignants en biologie. Au Canada, les médecins spécialistes forment la moitié des abonnés de la revue, les généralistes $25 \%$ et les étudiants $15 \%$. La diffusion de médecine/sciences hors des deux pays fondateurs reste limitée, mais progresse régulièrement, principalement en Belgique. Le questionnaire rempli par les lecteurs européens nous a permis d'avoir une idée des jugements d'ensemble sur la revue et du niveau "d'adéquation " entre elle et son lectorat. $70 \%$ des abonnés ayant répondu à ce questionnaire sont très satisfaits des rubriques articles de synthèse et nouvelles, $60 \%$ du lexique, 30 à $40 \%$ des hypothèses et débats, notes originales et éditoriaux; $56 \%$ souhaitent un développement des articles de synthèse et des nouvelles.

A la question: "à quel public médecine/sciences est-elle, selon vous, le mieux adaptée? ", nous avons eu $23 \%$ de réponses : aux étudiants, $29 \%$ aux hospitalo-universitaires, $37 \%$ aux chercheurs et $11 \%$ à tous les publics. Enfin, un même nombre d'abonnés souhaiterait que la revue devienne plus clinique et médicale... ou, au contraire, plus scientifique et fondamentale. Cette enquête constitue pour l'équipe de médecine/sciences un enseignement important et contient des motifs de satisfaction autant que d'insatisfaction. Il semble que notre positionnement entre la biologie et la médecine... "la biologie d'aujourd'hui et la médecine de demain ", comme le dit notre slogan, soit globalement correct puisque les indices de satisfaction sont très comparables parmi les lecteurs à dominante médicale et scientifique.

Nous avons cependant conscience de ne pas réussir toujours à tirer les leçons d'intérêt médical des découvertes fondamentales que nous annonçons et commentons et, symétriquement, de publier des articles médicaux totalement inintelligibles pour les non-médecins. Corriger en partie le premier défaut sera probablement plus aisé que de se défaire du second.

Les nouvelles, dont nous avons considérablement augmenté le nombre de pages, sont fort appréciées... ce dont nous nous réjouissons car il s'agit là d'une rubrique essentielle de médecine/sciences, probablement la plus originale, sans équivalent en langue française. Assez souvent ces nouvelles sont développées jusqu'à constituer de brèves revues d'actualité donnant les références essen- 
tielles les plus récentes sur un sujet. Les thèmes traités privilégient - peut-être trop - les disciplines biologiques, notamment la génétique moléculaire; mais il s'agit indiscutablement du domaine dont la progression est la plus éclatante à l'heure actuelle. Le jugement de nos lecteurs sur la forme actuelle des notes originales rejoint notre propre analyse que médecine/sciences apporte peu, en ce domaine, par rapport aux revues francophones préexistantes. Il est vrai que, là plus qu'ailleurs, intervient le rôle dominant de l'anglais dans la diffusion des résultats scientifiques; de plus, nous ne sommes pas encore cités dans le Current Contents (les démarches pour ce faire ne peuvent être entreprises qu'au terme de 18 mois de parution), ce qui amoindrit la diffusion des articles que nous publions. Quant à nos éditoriaux et à nos débats, ils devraient également trouver un ton plus juste. La fonction des éditoriaux est en effet d'ouvrir de nouvelles perspectives à partir des données apportées par des articles du numéro, plutôt que de simplement les résumer. Les débats devraient, quant à eux, traiter d'une question réellement controversée, devenant ainsi plus vivants.

Le lexique, enfin, est assez largement apprécié ; il nous semble un moyen essentiel de parvenir à notre but : démystifier la biologie moderne aux yeux de ceux qu'elle devrait naturellement intéresser mais qui sont effrayés par la terminologie obscure, par leur ignorance des concepts de base et la rapidité de l'évolution des connaissances. C'est tout naturellement que nous avons commencé à défricher ainsi le maquis du jargon et des techniques en génétique moléculaire, en abordant les bases théoriques, puis les applications à la médecine. Afin de permettre à chacun de consulter commodément ces lexiques, nous les réunirons, pour le volume 2 comme pour le volume 1 , en une brochure distribuée à nos abonnés ou vendue à l'unité.

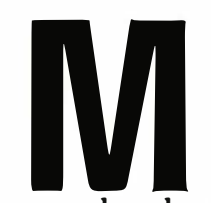

édecine/sciences : ce que nous voulons, ce que nous faisons. L'époque que nous vivons dans le monde de la biologie et de la médecine nous conduit à penser qu'il s'est créé une situation exceptionnellement propice à la communication entre ceux qui ont plus directement la charge de soigner, et les chercheurs de différentes disciplines. Les uns et les autres n'en ont pas toujours clairement conscience, mais ils sont bien les artisans d'une mêrne aventure, celle du décryptage progressif des langages de la vie, de ses désordres et de ses multiples dialectes.

Notre but, à médecine/sciences, est donc de témoigner de cette évolution, de l'accélérer si possible, d'y préparer les étudiants actuels. Notre "cible " est par conséquent double : les spécialistes de la santé de l'homme d'une part, l'ensemble des autres biologistes d'autre part. Pour que les uns et les autres trouvent un intérêt réciproque à leurs messages, il faut concilier la publication d'articles de très haut niveau scientifique... et leur intelligibilité pour tout lecteur curieux et cultivé. Il faut aussi s'efforcer de bien dégager chaque fois le message que l'on veut faire passer dans un article, les questions posées, les réponses ou hypothèses proposées et leurs implications au-delà du thème traité. C'est afin de parvenir à ou du moins de se rapprocher de - cet ambitieux objectif que tous les manuscrits soumis à médecine/sciences, qu'ils aient été sollicités ou non, sont revus en détail par deux lecteurs extérieurs informés du but poursuivi par la revue, puis par les membres du comité de rédaction. Pour ces derniers, l'un des critères requis est que l'article soit compris et ait intéressé celui d'entre nous qui est thématiquement le plus éloigné du sujet traité. Lorsqu'après correction par l'auteur, l'article est accepté, une ultime révision éditoriale permet enfin d'homogénéiser, s'il y a lieu, le style et de débusquer les termes techniques non définis.

Enfin, la plupart des schémas sont redessinés, parfois recomposés pour parvenir à la clarté maximum du message.

médecine/sciences, avons-nous dit, " doit être le lieu privilégié du dialogue entre médecine et sciences "... et nous souhaitons en effet développer ce dialogue sous des formes nouvelles : un courrier des lecteurs pourrait notamment permettre d'apporter des précisions sur un sujet traité, formuler des hypothèses, voire interpeller un auteur. Sous une forme plus élaborée, nous sommes disposés à publier des hypothèses pertinentes et, surtout, des débats présentant des vues différentes d'une même question. Enfin, comme nous l'avons déjà fait dans le volume 2 , nous sommes prêts à considérer la publication accélérée sur des sujets de très grande importance, de notes brèves (lettre $\dot{a} \mathrm{~m} / \mathrm{s}$, de 3 à 4000 signes).

Se donner les moyens pour notre revue de répondre correctement aux exigences de nos lecteurs, et à la nôtre propre, supposait que notre pagination soit augmentée... ce qui est déjà une réalité depuis plusieurs numéros puisque nous sommes passés de 48 à 56 pages rédactionnelles. Notre tarif d'abonnement n'ayant pas été modifié depuis notre création... et le nombre de nos pages augmentant, il est malheureusement certain (dures réalités économiques !) que le prix de l'abonnement augmentera en 1987. Il restera cependant dans des limites raisonnables afin que médecine/sciences demeure une revue peu onéreuse, car, nous ne le répéterons jamais assez, la condition de notre succès est qu'elle constitue un outil essentiel de la circulation des idées et des connaissances en biologie et en médecine, outil qu'il faudra posséder... individuellement.

A tous nos lecteurs que nous espérons fidèles et auxquels nous demandons d'aider à la diffusion de $\mathrm{m} / \mathrm{s}$, au nom de tous les membres des comités de rédaction, je souhaite une bonne année 